Copyright (C) 2014 IEEE. Personal use of this material is permitted. Permission from IEEE must be obtained for all other uses, in any current or future media, including reprinting/republishing this material for advertising or promotional purposes, creating new collective works, for resale or redistribution to servers or lists, or reuse of any copyrighted component of this work in other works. 


\title{
Two-way Compress-and-forward Relaying With Multiple MIMO Relay Nodes
}

\author{
Yue Rong, Senior Member, IEEE
}

\begin{abstract}
In this letter, we consider a two-way communication system where two source nodes exchange information via multiple relay nodes. The source and relay nodes may have multiple antennas and the compress-and-forward strategy is applied at each relay node. We aim at maximizing the sum-rate of two-way communication. An iterative algorithm is proposed to jointly optimize the source precoding matrices, the compression noise covariance matrices, and the time-sharing factor. Numerical simulation results show a better performance of the proposed algorithm compared with existing techniques.
\end{abstract}

Index Terms-Compress-and-forward, MIMO relay, two-way relay.

\section{INTRODUCTION}

Due to its great potential in extending the network coverage and enhancing the link reliability, wireless relay communications have attracted much research interest recently. In general, there are three types of relay strategies [1]: amplify-andforward (AF), decode-and-forward (DF), and compress-andforward $(\mathrm{CF})$. In the AF strategy, the relay node only amplifies (including a possible linear transformation) and retransmits its received signals. Therefore, in general the AF strategy has lower complexity and shorter processing delay than the DF and CF strategies. However, the AF relay strategy does not stop the noise propagation. In the DF strategy, the relay node first decodes the information from the received signal. Then, the relay node re-encodes the information and retransmits the encoded signals. Thus, the DF strategy stops noise propagation, but at the cost of higher complexity and longer processing delay. The CF strategy provides an effective complexity-performance tradeoff by compressing the received signals at the relay node before forwarding them to the destination node.

When nodes in a relay network have multiple transmit/receive dimensions, we call such system a multiple-input multiple-output (MIMO) relay system. Performance of various relay strategies in MIMO relay systems has been investigated in [2]. Recent progress on the optimization of AF MIMO relay systems has been summarized in [3]. The optimization of the transmit covariance matrices in DF MIMO relay systems has been addressed in [4].

In [5], a one-way CF MIMO relay system with multiple relay nodes has been studied, where the covariance matrices of the compression noise vectors are optimized. For a twoway CF MIMO relay system with one relay node, the optimal covariance matrix of the compression noise vector has been derived recently in [6]. It is shown in [6] that the CF strategy can improve the system spectral efficiency, especially when the relay node is close to one of the two source nodes.

Y. Rong is with the Dept. Electrical and Computer Engineering, Curtin University, Bentley, WA 6102, Australia (e-mail: y.rong@ curtin.edu.au).

This work is supported by the Australian Research Council's Discovery Projects funding scheme (DP140102131).
In this letter, we consider a two-way communication system where two source nodes exchange information via multiple relay nodes and the source and relay nodes may have multiple antennas. Such system has been addressed in [7] where the AF strategy is used at relay nodes and the two-way sum mean-squared error (MSE) of the signal waveform estimation is adopted as the design criterion. Different to [7], in this letter, the CF strategy is applied at each relay node, and we aim at maximizing the sum-rate of two-way communication. Compared with [5], [6], [8], and [9], we consider two-way MIMO relay systems with multiple relay nodes and develop an iterative algorithm to jointly optimize the source precoding matrices, the compression noise covariance matrices, and the time-sharing factor.

Interestingly, we show that the optimal source precoding matrices have a beamforming structure, and the proposed algorithm can determine the optimal number of data streams that should be transmitted by each source node in order to maximize the sum-rate of two-way communication. Moreover, we optimize the covariance matrix of the compression noise vector at each relay node iteratively by exploiting the matrix approximate joint diagonalization. We also optimize the time-sharing factor between two stages of two-way relay communication. Numerical simulation results show a better performance of the proposed algorithm compared with existing techniques.

\section{SySTEM MODEL}

We consider a two-way communication system where two nodes exchange information with the assistance of $K$ relay nodes. For simplicity, we assume that each source node has $N$ antennas, while each relay node is equipped with $L$ antennas. The algorithm developed in this letter can be straightforwardly generalized to relay systems where all nodes have different number of antennas. Using half-duplex relay nodes, the communication between two source nodes is completed in two stages. At the first stage, node $i$ transmits $\mathbf{x}_{i}=\mathbf{B}_{i} \mathbf{s}_{i}$ to all relay nodes for a time of $t$, where $\mathbf{s}_{i}$ is the $M_{i} \times 1$ informationcarrying source symbol vector, and $\mathbf{B}_{i}$ is the $N \times M_{i}$ source precoding matrix at node $i$. In general, there is $M_{i} \leq N$, and the approach of choosing the optimal $M_{i}$ will be introduced later on. We assume that $\mathrm{E}\left[\mathbf{s}_{i} \mathbf{s}_{i}^{H}\right]=\mathbf{I}_{M_{i}}, i=1,2$, where $\mathrm{E}[\cdot]$ stands for the statistical expectation, $(\cdot)^{H}$ denotes the matrix (vector) Hermitian transpose, and $\mathbf{I}_{n}$ is the $n \times n$ identity matrix. The received signal vector at the $k$ th relay node is

$$
\mathbf{y}_{r, k}=\mathbf{H}_{r 1, k} \mathbf{B}_{1} \mathbf{s}_{1}+\mathbf{H}_{r 2, k} \mathbf{B}_{2} \mathbf{s}_{2}+\mathbf{v}_{r, k}, \quad k=1, \cdots, K
$$

where $\mathbf{H}_{r i, k}, i=1,2, k=1, \cdots, K$, is the $L \times N$ MIMO channel from node $i$ to the $k$ th relay node, $\mathbf{v}_{r, k}$ is the additive white Gaussian noise (AWGN) vector at the $k$ th relay node. 
At the second stage, the relay nodes compress the received signal vectors as

$$
\hat{\mathbf{y}}_{r, k}=\mathbf{y}_{r, k}+\mathbf{q}_{k}, \quad k=1, \cdots, K
$$

where $\mathbf{q}_{k}$ is the compression noise vector at the $k$ th relay node with complex Gaussian distribution as $\mathcal{C N}\left(\mathbf{0}, \mathbf{C}_{k}\right)$. Then $\hat{\mathbf{y}}_{k}$ is encoded to $\mathbf{x}_{r, k}$ and broadcasted to nodes 1 and 2 for the remaining time of $1-t$. The received signal vector $\mathbf{y}_{i}$ at node $i$ can be written as

$$
\mathbf{y}_{i}=\sum_{k=1}^{K} \mathbf{H}_{i r, k} \mathbf{x}_{r, k}+\mathbf{v}_{i}, \quad i=1,2
$$

where $\mathbf{H}_{i r, k}$ is the $N \times L$ MIMO channel from the $k$ th relay node to node $i$ and $\mathbf{v}_{i}$ is the AWGN vector at node $i$. We assume that $\mathbf{v}_{r, k}, k=1, \cdots, K$, and $\mathbf{v}_{i}, i=1,2$, are independent and identically distributed (i.i.d.) complex circularly symmetric AWGN with zero mean and unit variance.

Let us introduce $R_{i}$ as the rate of source node $i, i=1,2$. According to [8], the achievable rate region of the twoway MIMO multi-relay communication system using the $\mathrm{CF}$ strategy is given by

$$
\begin{aligned}
& \quad R_{i} \leq t I\left(\mathbf{s}_{i} ; \hat{\mathbf{y}}_{r} \mid \mathbf{s}_{i}\right), \quad i=1,2 \\
& \text { s.t. }
\end{aligned}
$$

where $I(\cdot ; \cdot)$ denotes mutual information, $\hat{\mathbf{y}}_{r} \triangleq\left[\hat{\mathbf{y}}_{r, 1}^{T}, \cdots, \hat{\mathbf{y}}_{r, K}^{T}\right]^{T}$, $\mathbf{y}_{r} \triangleq\left[\mathbf{y}_{r, 1}^{T}, \cdots, \mathbf{y}_{r, K}^{T}\right]^{T}$, and $\mathbf{x}_{r} \triangleq\left[\mathbf{x}_{r, 1}^{T}, \cdots, \mathbf{x}_{r, K}^{T}\right]^{T}$. Here, $(\cdot)^{T}$ denotes matrix (vector) transpose, $i=2$ for $i=1$, and $\bar{i}=1$ for $i=2$. The constraints in (5) guarantee that the information carried in $\hat{\mathbf{y}}_{r}$ can be reliably transmitted to nodes 1 and 2 [8].

For the two-way relay system in (1)-(3), the mutual information terms in (4) and (5) can be written as

$$
\begin{gathered}
I\left(\mathbf{s}_{i} ; \hat{\mathbf{y}}_{r} \mid \mathbf{s}_{i}^{-}\right)=\log \left|\mathbf{I}_{N}+\mathbf{B}_{i}^{H} \sum_{k=1}^{K} \mathbf{H}_{r i, k}^{H}\left(\mathbf{I}_{L}+\mathbf{C}_{k}\right)^{-1} \mathbf{H}_{r i, k} \mathbf{B}_{i}\right|(6) \\
I\left(\mathbf{y}_{r} ; \hat{\mathbf{y}}_{r} \mid \mathbf{s}_{i}\right)=\log \left|\mathbf{I}_{K L}+\left(\mathbf{H}_{r i} \mathbf{B}_{i} \mathbf{B}_{\bar{i}}^{H} \mathbf{H}_{r \bar{i}}^{H}+\mathbf{I}_{K L}\right) \mathbf{C}^{-1}\right| \\
I\left(\mathbf{x}_{r} ; \mathbf{y}_{i}\right)=\log \left|\mathbf{I}_{L}+\sum_{k=1}^{K} \frac{P_{r, k}}{L} \mathbf{H}_{i r, k}^{H} \mathbf{H}_{i r, k}\right|
\end{gathered}
$$

where $P_{r, k}, k=1, \cdots, K$, is the transmission power available at the $k$ th relay node, $(\cdot)^{-1}$ and $|\cdot|$ denote matrix inversion and determinant, respectively, $\mathbf{H}_{r i} \triangleq\left[\mathbf{H}_{r i, 1}^{T}, \cdots, \mathbf{H}_{r i, K}^{T}\right]^{T}$, $i=1,2, \mathbf{C} \triangleq \operatorname{bd}\left[\mathbf{C}_{1}, \cdots, \mathbf{C}_{K}\right]$, and $\mathrm{bd}[\cdot]$ stands for a block diagonal matrix.

\section{Proposed Joint Source And Relay Design ALGORITHM}

In this section, we develop an iterative algorithm to maximize the sum-rate of two-way communication $R_{1}+R_{2}$ through jointly optimizing the source precoding matrices $\mathbf{B}_{1}$ and $\mathbf{B}_{2}$, the covariance matrices of the compression noise vectors $\mathbf{C}_{k}$, $k=1, \cdots, K$, and the time-sharing factor $t$. Using (6)-(8), the sum-rate optimization problem can be written as

$$
\begin{array}{rl}
\max _{t,\left\{\mathbf{C}_{k}\right\}, \mathbf{B}_{1}, \mathbf{B}_{2}} & t \sum_{\substack{i=1\\
}}^{2} \log \mid \mathbf{I}_{N}+\mathbf{B}_{i}^{H} \sum_{k=1}^{K} \mathbf{H}_{r i, k}^{H} \mathbf{C}_{k}^{-1}\left(\mathbf{I}_{L}+\mathbf{C}_{k}^{-1}\right)^{-1} \\
& \times \mathbf{H}_{r i, k} \mathbf{B}_{i} \mid
\end{array}
$$

$$
\begin{array}{ll}
\text { s.t. } & t \log \left|\mathbf{I}_{K L}+\left(\mathbf{H}_{r i} \mathbf{B}_{i} \mathbf{B}_{i}^{H} \mathbf{H}_{r i}^{H}+\mathbf{I}_{K L}\right) \mathbf{C}^{-1}\right| \\
& \leq(1-t) a_{i}, \quad i=1,2 \\
& \operatorname{tr}\left(\mathbf{B}_{i} \mathbf{B}_{i}^{H}\right) \leq P_{i}, \quad i=1,2 \\
& 0<t<1, \quad \mathbf{C}_{k} \geq 0, \quad k=1, \cdots, K
\end{array}
$$

where $\left\{\mathbf{C}_{k}\right\} \triangleq\left\{\mathbf{C}_{k}, k=1, \cdots, K\right\}, \operatorname{tr}(\cdot)$ denotes matrix trace, $P_{i}, i=1,2$, is the transmission power available at the $i$ th source node, and $a_{i} \triangleq \log \left|\mathbf{I}_{L}+\sum_{k=1}^{K} \frac{P_{r, k}}{L} \mathbf{H}_{i r, k}^{H} \mathbf{H}_{\bar{i} r, k}\right|$, $i=1,2$. It can be expected that the system sum-rate increases with the number of relay nodes.

The problem (9)-(12) is highly nonconvex with matrix variables, and the globally optimal solution is intractable to obtain. In the following, we develop an iterative algorithm to solve the problem (9)-(12). First, we show the optimal structure of $\mathbf{B}_{1}$ and $\mathbf{B}_{2}$.

\section{A. Optimal Source Precoding Matrices}

Note that since

$$
\begin{aligned}
& \left|\mathbf{I}_{K L}+\left(\mathbf{H}_{r i} \mathbf{B}_{i} \mathbf{B}_{i}^{H} \mathbf{H}_{r i}^{H}+\mathbf{I}_{K L}\right) \mathbf{C}^{-1}\right| \\
= & \left|\mathbf{I}_{K L}+\mathbf{C}+\mathbf{H}_{r i} \mathbf{B}_{i} \mathbf{B}_{i}^{H} \mathbf{H}_{r i}^{H}\right|\left|\mathbf{C}^{-1}\right| \\
= & \left|\mathbf{I}_{K L}+\mathbf{H}_{r i} \mathbf{B}_{i} \mathbf{B}_{i}^{H} \mathbf{H}_{r i}^{H}\left(\mathbf{I}_{K L}+\mathbf{C}\right)^{-1}\right|\left|\mathbf{C}^{-1}\right|\left|\mathbf{I}_{K L}+\mathbf{C}\right| \\
= & \left|\mathbf{I}_{M_{i}}+\mathbf{B}_{i}^{H} \sum_{k=1}^{K} \mathbf{H}_{r i, k}^{H} \mathbf{C}_{k}^{-1}\left(\mathbf{I}_{L}+\mathbf{C}_{k}^{-1}\right)^{-1} \mathbf{H}_{r i, k} \mathbf{B}_{i}\right| \\
& \times\left|\mathbf{I}_{K L}+\mathbf{C}^{-1}\right|, \quad i=1,2
\end{aligned}
$$

the constraints in (10) can be rewritten as

$$
\log \left|\mathbf{I}_{M_{i}}+\mathbf{B}_{i}^{H} \sum_{k=1}^{K} \mathbf{H}_{r i, k}^{H} \mathbf{C}_{k}^{-1}\left(\mathbf{I}_{L}+\mathbf{C}_{k}^{-1}\right)^{-1} \mathbf{H}_{r i, k} \mathbf{B}_{i}\right| \leq e_{i}
$$

where $e_{i} \triangleq a_{i}(1-t) / t-\log \left|\mathbf{I}_{K L}+\mathbf{C}^{-1}\right|, i=1,2$.

It can be seen from (9), (11), and (14) that for given $\left\{\mathbf{C}_{k}\right\}$ and $t, \mathbf{B}_{1}$ and $\mathbf{B}_{2}$ can be optimized separately through solving the problem of

$$
\begin{aligned}
& \max _{\mathbf{B}_{i}} \log \left|\mathbf{I}_{M_{i}}+\mathbf{B}_{i}^{H} \sum_{k=1}^{K} \mathbf{H}_{r i, k}^{H} \mathbf{C}_{k}^{-1}\left(\mathbf{I}_{L}+\mathbf{C}_{k}^{-1}\right)^{-1} \mathbf{H}_{r i, k} \mathbf{B}_{i}\right| \\
& \text { s.t. } \log \left|\mathbf{I}_{M_{i}}+\mathbf{B}_{i}^{H} \sum_{k=1}^{K} \mathbf{H}_{r i, k}^{H} \mathbf{C}_{k}^{-1}\left(\mathbf{I}_{L}+\mathbf{C}_{k}^{-1}\right)^{-1} \mathbf{H}_{r i, k} \mathbf{B}_{i}\right| \leq e_{2}(16) \\
& \quad \operatorname{tr}\left(\mathbf{B}_{i} \mathbf{B}_{i}^{H}\right) \leq P_{i} .
\end{aligned}
$$

Let us introduce the eigenvalue decomposition (EVD) of

$$
\sum_{k=1}^{K} \mathbf{H}_{r i, k}^{H} \mathbf{C}_{k}^{-1}\left(\mathbf{I}_{L}+\mathbf{C}_{k}^{-1}\right)^{-1} \mathbf{H}_{r i, k}=\mathbf{V}_{i} \boldsymbol{\Delta}_{i} \mathbf{V}_{i}^{H}, \quad i=1,2
$$

where $\mathbf{V}_{i}$ is the $N \times M_{i}$ (semi)-unitary eigenvector matrix with $\mathbf{V}_{i}^{H} \mathbf{V}_{i}=\mathbf{I}_{M_{i}}, \boldsymbol{\Delta}_{i}$ is the $M_{i} \times M_{i}$ eigenvalue matrix with the main diagonal elements sorted in descending order. It can be easily shown [9] that the optimal $\mathbf{B}_{i}$ as the solution to the problem (15)-(17) has the structure of

$$
\mathbf{B}_{i}=\mathbf{V}_{i} \Theta_{i}^{\frac{1}{2}}, \quad i=1,2
$$

where $\boldsymbol{\Theta}_{i}$ is an $M_{i} \times M_{i}$ diagonal matrix. In fact, $M_{i}$ is the rank of $\sum_{k=1}^{K} \mathbf{H}_{r i, k}^{H} \mathbf{C}_{k}^{-1}\left(\mathbf{I}_{L}+\mathbf{C}_{k}^{-1}\right)^{-1} \mathbf{H}_{r i, k}$. Thus, (18) 
determines the optimal number of data streams that should be transmitted by each source node in order to maximize the sum-rate of two-way communication.

Using (18), the problem (15)-(17) can be written as

$$
\begin{aligned}
\max _{\theta_{i, 1}, \cdots, \theta_{i, M_{i}}} & \sum_{n=1}^{M_{i}} \log \left(1+\delta_{i, n} \theta_{i, n}\right) \\
\text { s.t. } & \sum_{n=1}^{M_{i}} \log \left(1+\delta_{i, n} \theta_{i, n}\right) \leq e_{i} \\
& \sum_{n=1}^{M_{i}} \theta_{i, n} \leq P_{i}, \theta_{i, n} \geq 0, n=1, \cdots, M_{i}
\end{aligned}
$$

where $\delta_{i, n}$ and $\theta_{i, n}$ are the $n$th main diagonal elements of $\boldsymbol{\Delta}_{i}$ and $\boldsymbol{\Theta}_{i}$, respectively. Using the Lagrange multiplier method [11] to solve the problem (19)-(21), we obtain

$$
\theta_{i, n}=\left(1 / \nu_{i}-1 / \delta_{i, n}\right)^{+}, \quad n=1, \cdots, M_{i}
$$

where $x^{+} \triangleq(x, 0), \nu_{i}>0$ is the Lagrangian multiplier and can be found as follows. Let us assume that the constraint $\sum_{n=1}^{M_{i}} \theta_{i, n} \leq P_{i}$ is active at the optimal solution, then $\nu_{i}$ is the solution to the nonlinear equation of

$$
\sum_{n=1}^{M_{i}}\left(1 / \nu_{i}-1 / \delta_{i, n}\right)^{+}=P_{i}
$$

Otherwise, the constraint (20) must be active at the optimal point. Then $\nu_{i}$ is the solution to the nonlinear equation of

$$
\sum_{n=1}^{M_{i}} \log \left(1+\delta_{i, n}\left(1 / \nu_{i}-1 / \delta_{i, n}\right)^{+}\right)=e_{i} .
$$

Since the left-hand side of (22) and (23) are both monotonically decreasing with respect to $\nu_{i},(22)$ and (23) can be efficiently solved using the bisection method [11].

\section{B. Optimization of the Compression Noise Covariance Matri- ces and the Time-sharing Factor}

From (9), (10), (12), and (13), for fixed $\mathbf{B}_{1}, \mathbf{B}_{2}$, and $t$, $\left\{\mathbf{C}_{k}\right\}$ can be optimized by solving the problem of

$$
\begin{aligned}
& \max _{\left\{\mathbf{C}_{k}\right\}} \sum_{i=1}^{2} \log \left|\mathbf{I}_{M_{i}}+\sum_{k=1}^{K} \overline{\mathbf{H}}_{r i, k}^{H} \mathbf{C}_{k}^{-1}\left(\mathbf{I}_{L}+\mathbf{C}_{k}^{-1}\right)^{-1} \overline{\mathbf{H}}_{r i, k}\right| \\
& \text { s.t. } \log \left|\mathbf{I}_{M_{i}}+\sum_{k=1}^{K} \overline{\mathbf{H}}_{r i, k}^{H} \mathbf{C}_{k}^{-1}\left(\mathbf{I}_{L}+\mathbf{C}_{k}^{-1}\right)^{-1} \overline{\mathbf{H}}_{r i, k}\right| \\
& \quad+\log \left|\mathbf{I}_{K L}+\mathbf{C}^{-1}\right| \leq b_{i}, \quad i=1,2 \\
& \quad \mathbf{C}_{k} \geq 0, \quad k=1, \cdots, K
\end{aligned}
$$

where $\overline{\mathbf{H}}_{r i, k} \triangleq \mathbf{H}_{r i, k} \mathbf{B}_{i}, b_{i} \triangleq a_{i}(1-t) / t, i=1,2$. The problem (24)-(26) can be solved by updating $\left\{\mathbf{C}_{k}\right\}$ alternatingly. For fixed $\mathbf{C}_{j}, j=1, \cdots, K, j \neq k$, the problem of optimizing $\mathbf{C}_{k}$ can be written as

$$
\begin{aligned}
\max _{\mathbf{C}_{k}} & \sum_{i=1}^{2} \log \left|\mathbf{A}_{i, k}+\overline{\mathbf{H}}_{r i, k}^{H} \mathbf{C}_{k}^{-1}\left(\mathbf{I}_{L}+\mathbf{C}_{k}^{-1}\right)^{-1} \overline{\mathbf{H}}_{r i, k}\right| \\
\text { s.t. } & \log \left|\mathbf{A}_{i, k}+\overline{\mathbf{H}}_{r i, k}^{H} \mathbf{C}_{k}^{-1}\left(\mathbf{I}_{L}+\mathbf{C}_{k}^{-1}\right)^{-1} \overline{\mathbf{H}}_{r i, k}\right| \\
& +\log \left|\mathbf{I}_{L}+\mathbf{C}_{k}^{-1}\right| \leq c_{i, k}, \quad i=1,2 \\
& \mathbf{C}_{k} \geq 0
\end{aligned}
$$

where $\mathbf{A}_{i, k} \triangleq \mathbf{I}_{M_{i}}+\sum_{j=1, j \neq k}^{K} \overline{\mathbf{H}}_{r i, j}^{H} \mathbf{C}_{j}^{-1}\left(\mathbf{I}_{L}+\mathbf{C}_{j}^{-1}\right)^{-1} \overline{\mathbf{H}}_{r i, j}$, $c_{i, k} \triangleq b_{i}-\sum_{j=1, j \neq k}^{K} \log \left|\mathbf{I}_{L}+\mathbf{C}_{j}^{-1}\right|, i=1,2$. Note that by using the identity of

$$
\begin{aligned}
& \left|\mathbf{A}_{i, k}+\overline{\mathbf{H}}_{r i, k}^{H} \mathbf{C}_{k}^{-1}\left(\mathbf{I}_{L}+\mathbf{C}_{k}^{-1}\right)^{-1} \overline{\mathbf{H}}_{r i, k}\right| \\
= & \left|\mathbf{I}_{L}+\overline{\mathbf{H}}_{r i, k} \mathbf{A}_{i, k}^{-1} \overline{\mathbf{H}}_{r i, k}^{H} \mathbf{C}_{k}^{-1}\left(\mathbf{I}_{L}+\mathbf{C}_{k}^{-1}\right)^{-1}\right|\left|\mathbf{A}_{i, k}\right| \\
= & \left|\mathbf{I}_{L}+\left(\mathbf{I}_{L}+\overline{\mathbf{H}}_{r i, k} \mathbf{A}_{i, k}^{-1} \overline{\mathbf{H}}_{r i, k}^{H}\right) \mathbf{C}_{k}^{-1}\right|\left|\mathbf{I}_{L}+\mathbf{C}_{k}^{-1}\right|^{-1}\left|\mathbf{A}_{i, k}\right|
\end{aligned}
$$

the optimization problem (27)-(29) can be rewritten as

$$
\begin{aligned}
\max _{\mathbf{C}_{k}} & \sum_{i=1}^{2} \log \left|\mathbf{I}_{L}+\left(\mathbf{I}_{L}+\overline{\mathbf{H}}_{r i, k} \mathbf{A}_{i, k}^{-1} \overline{\mathbf{H}}_{r i, k}^{H}\right) \mathbf{C}_{k}^{-1}\right| \\
& -2 \log \left|\mathbf{I}_{L}+\mathbf{C}_{k}^{-1}\right| \\
\text { s.t. } & \log \left|\mathbf{I}_{L}+\left(\mathbf{I}_{L}+\overline{\mathbf{H}}_{r i, k} \mathbf{A}_{i, k}^{-1} \overline{\mathbf{H}}_{r i, k}^{H}\right) \mathbf{C}_{k}^{-1}\right| \leq d_{i, k}, i=1,2(31 \\
& \mathbf{C}_{k} \geq 0
\end{aligned}
$$

where $d_{i, k} \triangleq c_{i, k}-\log \left|\mathbf{A}_{i, k}\right|$.

Let us introduce the following approximate joint diagonalization [10] of two matrices

$$
\mathbf{I}_{L}+\overline{\mathbf{H}}_{r i, k} \mathbf{A}_{i, k}^{-1} \overline{\mathbf{H}}_{r i, k}^{H}=\mathbf{U}_{k} \boldsymbol{\Sigma}_{i, k} \mathbf{U}_{k}^{H}, \quad i=1,2
$$

where $\mathbf{U}_{k}$ is a unitary matrix and $\boldsymbol{\Sigma}_{i, k}, i=1,2$, are approximate diagonal matrices. By neglecting the off-diagonal elements of $\boldsymbol{\Sigma}_{i, k}, i=1,2$, the optimal $\mathbf{C}_{k}^{-1}$ is given by

$$
\mathbf{C}_{k}^{-1}=\mathbf{U}_{k} \boldsymbol{\Lambda}_{k} \mathbf{U}_{k}^{H}
$$

where $\boldsymbol{\Lambda}_{k}$ is a diagonal matrix. Based on (33) and (34), the problem (30)-(32) can be approximated as

$$
\begin{aligned}
\max _{\lambda_{k, 1}, \cdots, \lambda_{k, L}} & \sum_{l=1}^{L} \sum_{i=1}^{2}\left[\log \left(\sigma_{i, k, l} \lambda_{k, l}+1\right)-\log \left(\lambda_{k, l}+1\right)\right] \\
\text { s.t. } & \sum_{l=1}^{L} \log \left(\sigma_{i, k, l} \lambda_{k, l}+1\right) \leq d_{i, k}, \quad i=1,2 \\
& \lambda_{k, l} \geq 0, \quad l=1, \cdots, L
\end{aligned}
$$

where $\sigma_{i, k, l}$ and $\lambda_{k, l}, l=1, \cdots, L$, are the $l$ th main diagonal elements of $\boldsymbol{\Sigma}_{i, k}$ and $\boldsymbol{\Lambda}_{k}$, respectively. Using the Lagrange multiplier method [11], the solution to the problem (35)-(37) is given by

$$
\lambda_{k, l}=-g_{k, l, 1}+\sqrt{g_{k, l, 1}^{2}-g_{k, l, 2}}, \quad l=1, \cdots, L
$$

where

$$
\begin{aligned}
& g_{k, l, 1}=\frac{1}{2}\left[1+\frac{1}{\mu_{k, 1}+\mu_{k, 2}}\left(\frac{1+\mu_{k, 1}}{\sigma_{2, k, l}}+\frac{1+\mu_{k, 2}}{\sigma_{1, k, l}}-2\right)\right] \\
& g_{k, l, 2}=\frac{1}{\mu_{k, 1}+\mu_{k, 2}}\left(\frac{\mu_{k, 1}-1}{\sigma_{2, k, l}}+\frac{\mu_{k, 2}-1}{\sigma_{1, k, l}}+\frac{2}{\sigma_{1, k, l} \sigma_{2, k, l}}\right) .
\end{aligned}
$$

Here $\mu_{k, 1} \geq 0$ and $\mu_{k, 2} \geq 0$ are the Lagrangian multipliers. We would like to mention that the approximate joint diagonalization approach has been used in [6] to optimize the compression noise covariance matrix for a two-way CF MIMO relay system with a single relay node.

For given $\mathbf{B}_{1}, \mathbf{B}_{2}$, and $\mathbf{C}$, based on the constraint (10), the optimal $t$ is given by

$$
t=\min _{i=1,2} \frac{a_{i}}{\log \left|\mathbf{I}_{K L}+\left(\mathbf{H}_{r i} \mathbf{B}_{i} \mathbf{B}_{i}^{H} \mathbf{H}_{r i}^{H}+\mathbf{I}_{K L}\right) \mathbf{C}^{-1}\right|+a_{i}}
$$


TABLE I

PROCEDURE OF SOLVING THE PROBLEM (9)-(12) BY THE PROPOSED ITERATIVE ALGORITHM

1) Initialize the algorithm with $\mathbf{C}_{k}^{(0)}=\mathbf{I}_{L}, k=1, \cdots, K$, and $\mathbf{B}_{i}^{(0)}=$ $\sqrt{P_{i} / N} \mathbf{I}_{N}, i=1,2$; Set $m=0$.

2) Update $t^{(m+1)}$ as (39) with fixed $\left\{\mathbf{C}_{k}^{(m)}\right\}$ and $\left\{\mathbf{B}_{i}^{(m)}\right\}$.

3) For $i=1,2$, update $\mathbf{B}_{i}^{(m+1)}$ as (18) through solving the problem (19)-(21) with given $\left\{\mathbf{C}_{k}^{(m)}\right\}$ and $t^{(m+1)}$.

4) For $k=1, \cdots, K$, update $\mathbf{C}_{k}^{(m+1)}$ as (34) by solving the problem (35)-(37) with fixed $\left\{\mathbf{B}_{i}^{(m+1)}\right\}, t^{(m+1)}$, and $\mathbf{C}_{j}^{(m)}, j=1, \cdots, L$, $j \neq k$.

5) If $\mathrm{SR}^{(m+1)}-\mathrm{SR}^{(m)} \leq \varepsilon$, then end.

Otherwise, let $m:=m+1$ and go to Step 2 .

Obviously, $t$ given by (39) satisfies $0<t<1$.

The procedure of using the proposed iterative algorithm to solve the problem (9)-(12) is listed on Table I, where the superscript $(m)$ stands for the variable in the $m$ th iteration, SR refers to the sum-rate (9), and $\varepsilon$ is a small positive number of convergence criterion. We observed that under $\varepsilon=0.01$, the proposed algorithm usually converges within 10 iterations, although a rigorous proof of the convergence is difficult. Since the complexity order of updating $\mathbf{B}_{i}$ and $\mathbf{C}_{k}$ is $\mathcal{O}\left(N^{3}\right)$ and $\mathcal{O}\left(L^{2}\right)$, respectively, the per-iteration computational complexity of the proposed algorithm is $\mathcal{O}\left(N^{3}+K L^{2}\right)$.

\section{NUMERICAL EXAMPLES}

In the numerical simulations, all channel matrices have i.i.d. complex Gaussian entries with zero-mean and unit variance. The noises are i.i.d. Gaussian with zero mean and unit variance. We assume that $P_{r, k}=P_{r}, k=1, \cdots, K$, and set $P_{1}=P_{2}=0 \mathrm{~dB}$.

In the first example, we set $K=1, N=L=4$, and compare the performance of the proposed algorithm with (a) the "Relay Only" algorithm in [6] where the compression noise covariance matrix $\mathbf{C}_{1}$ is optimized based on $\mathbf{B}_{i}=\sqrt{P_{i} / N} \mathbf{I}_{N}$, $i=1,2$, and $t=0.5$; (b) the "Source and Relay Only" algorithm where $\mathbf{C}_{1}, \mathbf{B}_{1}$, and $\mathbf{B}_{2}$ are optimized iteratively with $t=0.5$. The sum-rate of three algorithms versus $P_{r}$ is shown in Fig. 1. It can be seen that the Relay Only algorithm has the lowest sum-rate as the source precoding matrices and the time-sharing factor are not optimized. By optimizing the source precoding matrices, the system sum-rate can be greatly improved. Obviously, the proposed algorithm yields the largest sum-rate as it jointly optimizes $\mathbf{C}_{1}, \mathbf{B}_{1}, \mathbf{B}_{2}$, and $t$. Interestingly, we observed that the optimal $t$ increases with $P_{r}$, and at the low $P_{r}$ range $(1 \mathrm{~dB}-6 \mathrm{~dB})$, the optimal $t$ is between 0.45 and 0.55 . Therefore, in this example, $t=0.5$ is nearly optimal at low $P_{r}$.

In the second example, we study the sum-rate performance of the proposed algorithm for two-way MIMO relay systems with different $K, N$, and $L$. It can be clearly seen from Fig. 2 that as expected, the system sum-rate increases with the number of relay nodes and the number of antennas. We can also observe that the proposed CF two-way MIMO relay system has a higher sum-rate than the AF relay system in [7].

\section{CONCLUSiON}

We have developed an iterative algorithm to jointly optimize the source precoding matrices, the compression noise

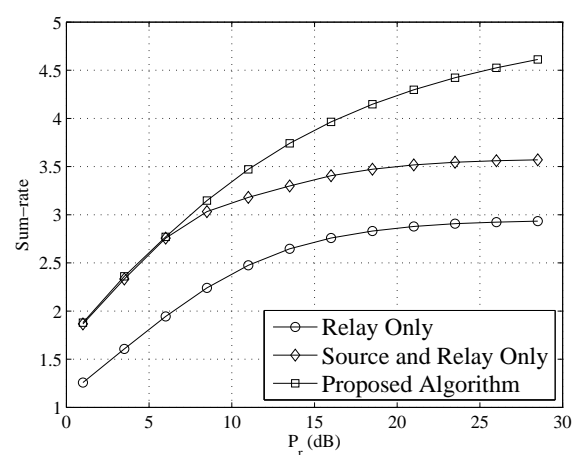

Fig. 1. Sum-rate of different algorithms versus $P_{r}$ with $K=1$.

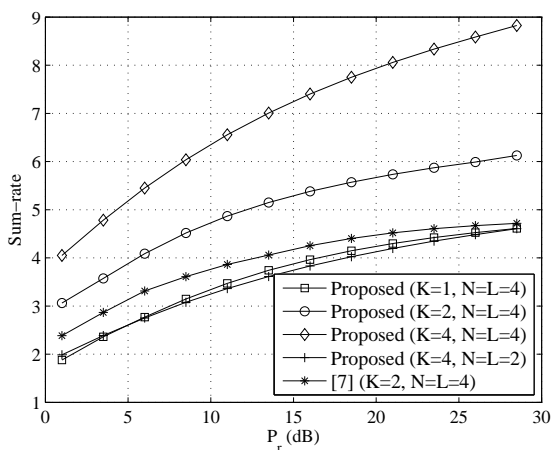

Fig. 2. Sum-rate versus $P_{r}$ with different $K, N, L$.

covariance matrices, and the time-sharing factor of two-way MIMO CF relay systems with multiple relay nodes. Simulation results show a better performance of the proposed algorithm compared with existing methods.

\section{REFERENCES}

[1] G. Kramer, M. Gastpar, and P. Gupta, "Cooperative strategies and capacity theorems for relay networks," IEEE Trans. Inf. Theory, vol. 51, pp. 3037-3063, Sep. 2005.

[2] Y. Fan and J. Thompson, "MIMO configurations for relay channels: Theory and practice," IEEE Trans. Wireless Commun., vol. 6, pp. 17741786, May 2007.

[3] L. Sanguinetti, A. A. D'Amico, and Y. Rong, "A tutorial on the optimization of amplify-and-forward MIMO relay systems," IEEE $J$. Selet. Areas Commun., vol. 30, pp. 1331-1346, Sep. 2012.

[4] S. Simoens, O. Muñoz-Medina, J. Vidal, and A. del Coso, "On the Gaussian MIMO relay channel with full channel state information," IEEE Trans. Signal Process. vol. 57, pp. 3588-3599, Sep. 2009.

[5] A. del Coso and S. Simoens, "Distributed compression for MIMO coordinated networks with a backhaul constraint," IEEE Trans. Wireless Commun., vol. 8, pp. 4698-4709, Sep. 2009.

[6] X. Lin, M. Tao, and Y. Xu, "MIMO two-way compress-and-forward relaying with approximate joint eigen-decomposition," IEEE Commun. Lett., vol. 17, pp. 1750-1753, Sep. 2013.

[7] Y. Rong, "Joint source and relay optimization for two-way MIMO multi-relay networks," IEEE Commun. Lett., vol. 15, pp. 1329-1331, Dec. 2011.

[8] S. J. Kim, N. Devroye, P. Mitran, and V. Tarokh, "Achievable rate regions and performance comparison of half duplex bi-directional relaying protocols," IEEE Trans. Inf. Theory, vol. 57, pp. 6405-6418, Oct. 2011.

[9] D. P. Palomar, J. M. Cioffi, and M. A. Lagunas, "Joint Tx-Rx beamforming design for multicarrier MIMO channels: A unified framework for convex optimization," IEEE Trans. Signal Process., vol. 51, pp. 23812401, Sep. 2003.

[10] J.-F. Cardoso and A. Souloumiac, "Jacobi angles for simultaneous diagonalization," SIAM Journal of Matrix Analysis and Applications, vol. 17, pp. 161-164, Jan. 1996.

[11] S. Boyd and L. Vandenberghe, Convex Optimization, Cambridge University Press, 2004. 